\title{
Gravitational Binding Energy in Charged Cylindrical Symmetry
}

\author{
M. Sharif *and M. Zaeem Ul Haq Bhatti ${ }^{\dagger}$ \\ Department of Mathematics, University of the Punjab, \\ Quaid-e-Azam Campus, Lahore-54590, Pakistan.
}

\begin{abstract}
We consider static cylindrically symmetric charged gravitating object with perfect fluid and investigate the gravitational binding energy. It is found that only the localized part of the mass function provides the gravitational binding energy, whereas the non-localized part generated by the electric coupling does not contribute for such energy.
\end{abstract}

Keywords: Relativistic fluids; Electromagnetic field; Cylindrically symmetric system; Binding energy.

PACS: 04.40.Nr; 41.20.-q; 04.20.Cv.

\section{Introduction}

In relativistic astrophysics, the energy required to break up the body into space is the gravitational binding energy which is found to be equal to the negative of the gravitational potential energy under certain conditions. In general relativity, different forms of mass energy are mixed with gravitational binding energy in implicit ways that clearly show the relation between binding energy and mass function. As

$$
\text { Binding energy }=\text { mass change } \times c^{2}
$$

*msharif.math@pu.edu.pk

†mzaeem.math@gmail.com 
If we remove the binding energy from the system, it would cause the removal of mass.

The concept of binding energy is useful for understanding a gravitational system and also has its importance in the analysis of systems consisting of charged particles. Gravitational binding energy of a celestial body is the energy required to expand the material to infinity. In general relativity, gravitational binding energy might stabilize a pure electromagnetic object. For an object to be bound gravitationally, it must contain enough perfect fluid to generate binding energy which is sufficient to overcome the electrostatic repulsion.

The self-gravitating energy of a compact object emerges due to its selfinteractions. Misner and Sharp [1] argued that energy is localized in spherical system. Bondi [2] favored this opinion and provided the mass of cylindrical systems. Herrera and Di Prisco [3] found an explicit expression for the active gravitational mass which has a parameter established through the composition of relaxation time, energy density, temperature and pressure. Zhang et al. 4] explored the effect of charge on the critical mass of static spherical system and found that the presence of net charge does not effect the existence of critical mass. Herrera et al. [5] evaluated the mass function for the cylindrical system based on the spherically symmetric case and showed that how the thermoinertial effects reduce the inertial mass. Recently, we have found the mass function for the charged cylindrical system and expressed it in terms of structure scalars [6].

Sharif and his collaborators [7] investigated the effects of electromagnetic field on different aspects of static and non-static symmetric models. Tiwari et al. 8] proved that the mass energy density and pressure of the fluid have the electromagnetic origin. Using inertial mass with Lorentz approach, Gautreau [9] provided the correspondence of the electron mass with its gravitational mass in spherical symmetry. Ohanian [10] proved that the gravitational and inertial masses are equal for any arbitrary system of matter and gravitational field under certain conditions.

Recently, Corne and his collaborators [11] have investigated the electric coupling and gravitational binding of spherically symmetric charged objects for both isotropic and anisotropic fluids. This paper investigates charged isotropic perfect fluid in the cylindrical symmetry. We evaluate total mass of a charged object and show that the gravitational binding energy is completely destroyed if the mass density is removed from the fluid. 


\section{Cylindrical Symmetry and Electromagnetic Field}

The static cylindrically symmetric spacetime has the following form [12

$$
d s^{2}=-A^{2} d t^{2}+B^{2} d r^{2}+C^{2} d \theta^{2}+d z^{2} .
$$

Here, $A$ and $B$ are dimensionless while $C$ has the dimension of $r$. In order to preserve the cylindrical symmetry, we assume the following constraints

$$
-\infty \leq t \leq \infty, \quad 0 \leq r \leq \infty, \quad 0 \leq \theta \leq 2 \pi, \quad-\infty<z<\infty,
$$

on the coordinates. Also, we consider the collapsing cylinder filled with the charged perfect fluid whose energy-momentum tensor is defined as

$$
T_{\alpha \beta}=(\mu+P) V_{\alpha} V_{\beta}+P g_{\alpha \beta}+\frac{1}{4 \pi}\left(F_{\alpha}^{\gamma} F_{\beta \gamma}-\frac{1}{4} F_{\gamma \delta} F^{\gamma \delta} g_{\alpha \beta}\right),
$$

where $\mu, P$ and $V^{\alpha}$ are the energy density, pressure and four velocity of the fluid, respectively and $F_{\alpha \beta}$ is the Maxwell field tensor. In comoving coordinates, we have $V_{\alpha}=-A \delta_{\alpha}^{0}$ satisfying $V^{\alpha} V_{\alpha}=-1$.

The 00-component of the Einstein tensor is

$$
G_{00}=\left(\frac{A}{B}\right)^{2}\left(\frac{C^{\prime \prime}}{C}-\frac{B^{\prime} C^{\prime}}{B C}\right) .
$$

The electric field $\mathbf{E}=E(r) \mathbf{e}_{\mathbf{r}}$ is a physical field (radial electric field), where

$\mathbf{e}_{\mathbf{r}}$ is the unit normal vector in the radial direction. The 00-component of the energy-momentum tensor in electromagnetic field is

$$
T_{00}=\left(\mu+\frac{E^{2}}{8 \pi}\right) A^{2},
$$

where $F^{0 r}$ is taken to be the radial electric field $E^{r}$. Using Eqs.(33) and (44), we can write the 00-component of the Einstein-Maxwell field equation, $G_{\alpha \beta}=8 \pi T_{\alpha \beta}$, as

$$
\left(\frac{1}{B}\right)^{2}\left(\frac{C^{\prime \prime}}{C}-\frac{B^{\prime} C^{\prime}}{B C}\right)=8 \pi \mu+E^{2} .
$$


The electric field is determined by the charge distribution through Gauss law

$$
\nabla \cdot \mathbf{E}=4 \pi \rho
$$

where $\rho$ is the charge density.

To calculate the electric field inside the star, we use divergence of the electromagnetic field tensor $F_{; \nu}^{\mu \nu}=4 \pi J^{\mu}$, which implies that $E_{; r}^{r}=F_{; r}^{0 r}=$ $4 \pi J^{0}$, where

$$
F_{; r}^{0 r}=\frac{1}{\sqrt{-g}}\left[\sqrt{-g} F^{0 r}\right]_{, r} .
$$

Also, $J^{\mu}$ is the four current whose non-zero component is $J^{0}=\rho(r) V^{0}=$ $\rho(r) A^{-1}$. Thus we have

$$
F_{; r}^{0 r}=\frac{1}{\sqrt{-g}}\left[\sqrt{-g} F^{0 r}\right]_{, r}=4 \pi \rho A^{-1},
$$

where $\sqrt{-g}=A B C$. Also,

$$
\frac{1}{\sqrt{-g}}\left[\sqrt{-g} F^{0 r}\right]_{, r}=\frac{1}{A B C}\left[A B C F^{0 r}\right]_{, r}=\frac{1}{A B C}\left[C F^{\hat{0} \hat{r}}\right]_{, r},
$$

where hat indicates unit vector. Equations (7) and (8) lead to

$$
\frac{d}{d r}\left[C F^{\hat{0} \hat{r}}\right]=4 \pi \rho B C .
$$

Integration of this equation yields

$$
C F^{\hat{0} \hat{r}}=4 \pi \int_{0}^{r} \rho B C d r
$$

which can be written as

$$
C F^{\hat{0} \hat{r}}=q(r),
$$

where

$$
q(r)=4 \pi \int_{0}^{r} \rho B C d r
$$

is the charge contained in the coordinate system of radius $r$ [13]. We can also write Eq.(10) as

$$
F^{\hat{0} \hat{r}}=\frac{q(r)}{C}=E(r) .
$$




\section{Mass Function}

Thorne [14] defined C-energy as the gravitational mass per unit specific length of the cylinder given by

$$
E(r)=\tilde{m}(r)=\frac{1}{8}\left(1-l^{-2} \nabla^{\alpha} \tilde{r} \nabla_{\alpha} \tilde{r}\right),
$$

where $\varrho, l$ and $\tilde{r}$ are the circumference radius, specific length and areal radius, respectively, which satisfy

$$
\varrho^{2}=\xi_{(1) a} \xi_{(1)}^{a}, \quad l^{2}=\xi_{(2) a} \xi_{(2)}^{a}, \quad \tilde{r}=\varrho l .
$$

Here, $\xi_{(1)}=\frac{\partial}{\partial \theta}$ and $\xi_{(2)}=\frac{\partial}{\partial z}$ are the Killing vectors in cylindrical system. The specific energy of the cylinder in the interior region is written as

$$
\tilde{m}(r)=\frac{1}{8}\left[1-\left(\frac{C^{\prime}}{B}\right)^{2}\right] .
$$

Differentiating this equation with respect to $r$, we obtain

$$
\frac{d \tilde{m}}{d r}=-\frac{C C^{\prime}}{4}\left[\frac{C^{\prime \prime}}{B^{2} C}-\frac{B^{\prime} C^{\prime}}{B^{3} C}\right] .
$$

Using Eqs.(15) and (12) in (14), it follows that

$$
\frac{d \tilde{m}}{d r}=-\left[2 \pi \mu C C^{\prime}+\frac{q^{2} C^{\prime}}{4 C}\right] .
$$

The densities $\mu(r)$ and $\rho(r)$ vanish at the boundary of the star $r=R$, while the external region of the cylinder, $r \geqslant R$, provides the constant charge $q(r)$

$$
q(r)=q(R)=Q=4 \pi \int_{0}^{R} \rho B C d r .
$$

For $\mu(r)=0$ and $q(r)=Q$, Eq.(15) becomes

$$
\frac{d \tilde{m}}{d r}=-\frac{Q^{2} C^{\prime}}{4 C}
$$

after integration, it turns out to be

$$
\tilde{m}(r)=-\frac{Q^{2}}{4} \ln (C N),
$$


where $N$ is any arbitrary integration constant with dimension of $1 / L$. Also, Eq.(13) yields

$$
B^{2}=\frac{C^{\prime 2}}{1-8 \tilde{m}}=\frac{C^{2}}{1+2 Q^{2} \ln (C N)} .
$$

We define a new function $m(r)$

$$
\tilde{m}(r)=m(r)-\frac{q^{2}(r) \ln (C N)}{4},
$$

which leads Eq.(15) to

$$
\frac{d}{d r}\left(m(r)-\frac{q^{2}(r) \ln (C N)}{4}\right)=-\left[2 \pi \mu C C^{\prime}+\frac{q^{2} C^{\prime}}{4 C}\right] .
$$

Outside the star, this equation has total correspondence with our previous assumptions and provides $m(r)=m(R)=$ constant for $r \geqslant R$. However, inside the star, energy density is not zero and also charge is not constant. Thus Eq.(11) yields

$$
\frac{d q}{d r}=4 \pi \rho B C
$$

Using Eq.(21) in (22), it follows that

$$
\frac{d m}{d r}=-\left(2 \pi \mu C C^{\prime}-2 \pi \rho B C q \ln (C N)\right) .
$$

Integrating this equation, we obtain

$$
m(r)=-2 \pi \int_{0}^{r} \mu C C^{\prime} d r+2 \pi \int_{0}^{r} q(r) \rho(r) B C \ln (C N) d r
$$

which is called the mass function. Since the total energy is not localizable in general relativity, so it cannot be interpreted as the mass energy inside $r$. The expression

$$
M=m(R)=-\int_{0}^{R} 2 \pi \mu C C^{\prime} d r+\int_{0}^{R} 2 \pi q(r) \rho(r) B C \ln (C N) d r
$$

provides the total gravitational mass of the star. The second term of this expression shows the electromagnetic contribution in the system, called the electromagnetic mass of the star. Moreover, this corresponds to an integral over the proper volume, whereas the first integral does not have such correspondence. 
To make the first term as the integral over the proper volume, we rewrite $M$ in the form

$$
M=m(R)=-\int_{0}^{R} B^{-1} 2 \pi \mu B C C^{\prime} d r+\int_{0}^{R} 2 \pi q(r) \rho(r) B C \ln (C N) d r,
$$

where the integration in both terms is taken over the proper volume. Based on the analysis of the Keplerian motion of neutral test particles around the star, the above equation identifies the total gravitational mass of the star (similar to the electromagnetic mass of an electron in the Lorentz theory). The first term contains the factor

$$
B^{-1}=\left(\frac{1-8 \tilde{m}}{C^{\prime 2}}\right)^{\frac{1}{2}}=\left(\frac{1+2 q^{2} \ln (C N)}{C^{\prime 2}}\right)^{\frac{1}{2}},
$$

which represents the contribution of the gravitational binding energy to the mass of the charged object. Equation (23) can be rewritten as

$$
\begin{aligned}
M & =-\int_{0}^{R} 2 \pi \mu B C C^{\prime} d r-\int_{0}^{R} 2 \pi q(r) \rho(r) B C \ln (C N) d r \\
& +\int_{0}^{R}\left(1-B^{-1}\right) 2 \pi \mu B C C^{\prime} d r
\end{aligned}
$$

This shows that the gravity binds only the localized part of the mass, while it has no contribution for the non-localized part caused by the electric coupling. Here the integral

$$
\int_{0}^{R}\left(1-B^{-1}\right) 2 \pi \mu B C C^{\prime} d r
$$

is the gravitational binding energy of the object. We see that the gravitational binding energy is affected by the electric charge through its contribution in $\tilde{m}$. Moreover, we note that the cylindrical mass has the contribution of electric field from outside the cylinder as energy transfer may occur from infinity in the axial direction.

\section{Conclusions}

We have investigated the mass function by using the Einstein-Maxwell field equations in cylindrical system with perfect fluid. This electromagnetic mass 
contains all the properties of the inertial mass and have localized and nonlocalized interactions. Also, the gravity holds only the localized part but does not hold the non-localized part generated by the electric coupling. When $q(r)=0$ or $\rho=0$, the integral might become negative (23). In this case, gravity cannot hold the object together and hence the gravitational binding energy is completely destroyed.

\section{References}

[1] Misner, C.W. and Sharp, D.H.: Phys. Rev. B136(1964)571.

[2] Bondi, H.: Proc. Roy. Soc. London A427(1990)249.

[3] Herrera, L. and Di Prisco, A.: Gen. Relativ. Gravit. 31(1999)301.

[4] Zhang, J.L., Chau, W.Y. and Deng, T.Y.: Astrophys. Space Sci. 88(1982)81.

[5] Herrera, L., Di Prisco, A., Ospino, J. and Ibáñez.: Gen. Relativ. Gravit. 44(2012)2645.

[6] Sharif, M. and Bhatti, M.Z.: Gen. Relativ. Gravit. 44(2012)2811.

[7] Sharif, M. and Ismat, H.F.: Can. J. Phys. 89(2011)1203; Sharif, M. and Abass, G.: Astrophys. Space Sci. 335(2011)515; Sharif, M. and Siddiqa, A.: Gen. Relativ. Gravit. 43(2011)73; Sharif, M. and Azam, M.: Gen. Relativ. Gravit. 44(2012)1181.

[8] Tiwari, R.N., Rao, J.R. and Kanakamedala, R.R.: Phys. Rev. D30(1984) 489 .

[9] Gautreau, R.: Phys. Rev. D31(1985)1860.

[10] Ohanian, H.C.: arXiv:1010.5557.

[11] Corne, M., Kheyfets, A. and Miller, W.A.: Class. Quantum Grav. 24(2007)5999; Corne, M., Kheyfets, A., Piasio, J. and Voegele, C.: Int. J. Theor. Phys. 50(2011)2737.

[12] Sharif, M. and Azam, M.: JCAP 02(2012)043. 
[13] Sharif, M. and Fatima, S.: Gen. Relativ. Gravit. 43(2011)127.

[14] Thorne, K.S.: Phys. Rev. B138(1965)251. 